\title{
Nanostructural origin of giant Rashba effect in intercalated graphene
}

\author{
M. Krivenkov ${ }^{1,2}$, E. Golias ${ }^{1}$, D. Marchenko ${ }^{1}$, J. \\ Sánchez-Barriga ${ }^{1}$, G. Bihlmayer ${ }^{3}$, O. Rader $^{1}$ and A. Varykhalov ${ }^{1}$ \\ 1 Helmholtz-Zentrum Berlin für Materialien und Energie, \\ Elektronenspeicherring BESSY II, Albert-Einstein-Strasse 15, 12489 Berlin, Germany \\ 2 Institut für Physik und Astronomie, Universität Potsdam, \\ Karl-Liebknecht-Str. 24/25, 14476 Potsdam, Germany and \\ 3 Peter Grünberg Institut and Institute for Advanced Simulation, \\ Forschungszentrum Jülich and JARA, 52425 Jülich, Germany
}

\begin{abstract}
To enhance the spin-orbit interaction in graphene by a proximity effect without compromising the quasi-free-standing dispersion of the Dirac cones means balancing the opposing demands for strong and weak graphene-substrate interaction. So far, only the intercalation of Au under graphene/Ni(111) has proven successful which is unexpected since graphene prefers a large separation $(\sim 3.3 \AA)$ from a Au monolayer in equilibrium. Here, we investigate this system and find the solution in a nanoscale effect. We reveal that the Au largely intercalates as nanoclusters. Our density functional theory calculations show that the graphene is periodically stapled to the Ni substrate and this attraction presses graphene and Au nanoclusters together. This, in turn, causes a Rashba effect of the giant magnitude observed in experiment. Our findings show that nanopatterning of the substrate can be efficiently used for engineering of spin-orbit effects in graphene.
\end{abstract}

PACS numbers: 73.22.Pr, 75.70.Tj, 81.05.ue 


\section{INTRODUCTION}

Bringing graphene in epitaxial contact to metals delivers a versatile tunability of its structural, electronic and magnetic properties [1-3]. The graphene-metal interfaces have evolved as fruitful test grounds for various physical effects allowing to control quantum-size effects [4-7], charge doping, and band gap [8-12] as well as the spin texture of graphene [13-17].

Epitaxial graphene is grown on metal surfaces either by segregation of carbon from the bulk of the substrate [18-20] or, more commonly, by chemical vapour deposition (CVD), i.e., cracking of hydrocarbons at the hot metal surface. The CVD synthesis of graphene has been thoroughly investigated on the different faces of $\mathrm{Ni}[21-24]$ and $\mathrm{Co}[16,25,26]$. Recently, it was also achieved on Fe substrates [27, 28]. Other substrates on which CVD growth of graphene is possible are $\mathrm{Ir}[4,6,29], \mathrm{Ru}[30,31]$ and $\mathrm{Pt}[32]$.

The batch of crystalline metallic substrates supporting CVD growth of graphene is not very large. An effective way to extend the variety of interfaces is the intercalation of a foreign chemical species under the graphene after its synthesis, especially for those species which do not directly support CVD growth.

Prominent examples are monolayers of noble metals $\mathrm{Cu}, \mathrm{Ag}$, and $\mathrm{Au}$ intercalated under graphene on $\mathrm{Ni}(111)$ [10, 12, 22], rare earth monolayers [33], alkali metals [24, 34], semiconductors Si [35, 36] and Ge [37] and even fullerene molecules [38]. All these elements bring along particular effects on sign and strength of charge doping and the presence and size of a band gap in the Dirac cone.

Interfaces to certain materials can induce spin textures in graphene. The intrinsic spinorbit coupling in graphene is small[39, 40] as well as the density of states at the Fermi-level. Therefore, magnetic properties can only be induced extrinsically either through contact with ferromagnetic materials, inducing exchange-type spin polarization of the Dirac cone [16, 17] or through contact with high- $Z$ materials ( $Z$ denotes the atomic number) which cause a Rashba-type spin-splitting of the Dirac cone [15, 41-43]. The high- $Z$ materials impose the Rashba effect on graphene through electronic hybridization which can directly be observed in the band structure $[15,44,45]$. While ferromagnetic proximity of graphene has early on been observed [46], the advantage of the proximity spin-orbit effects is that they support spintronics applications of graphene that do not require ferromagnets or magnetic field. 
Recently a spin-orbit splitting of $17 \mathrm{meV}$ was demonstrated in transport measurements in graphene transferred onto $\mathrm{WS}_{2}[47]$.

For epitaxial graphene on metals, there are only a few experimental realizations of large induced spin-orbit splitting in the Dirac cone. At first, quasi-free-standing Dirac cone, predicted by calculations in graphene/Au(111) [8], was experimentally observed in $\mathrm{Au}-$ intercalated graphene/ $\mathrm{Ni}(111)$, where a $13 \mathrm{meV}$ spin splitting of the Dirac cone was detected [48]. Subsequent optimization of the sample preparation procedure yielded a giant $(\sim 100$ meV) splitting which could be explained in terms of a Rashba effect induced by hybridization between the graphene $\pi$-band and $5 d$ states of $\mathrm{Au}[15,44]$. In particular, the $\sim 100 \mathrm{meV}$ splitting does not occur when $\mathrm{Au}$ is replaced by the sp metal Bi [44]. Subsequently, comparable magnitudes of the spin-orbit splitting have been achieved for graphene on $\operatorname{Ir}(111)$. In that system, the spin-orbit splitting could be tuned by a factor of two by rotation of the graphene relative to the Ir [29]. Most recently, we have investigated the intercalation of $\mathrm{Au}$ into one-dimensionally corrugated graphene/Fe(110) [49]. We found in addition to a giant Rashba effect also an exotic hedgehog spin texture in the gapped Dirac cone [49] which confirmed an earlier theoretical prediction [50].

To date, the structural origin of the giant $(\sim 100 \mathrm{meV})$ spin splitting of the Dirac cone in Au-intercalated graphene on $\mathrm{Ni}(111)$ [15] has remained unclear. Figure 1 shows the dilemma. The Au monolayer [Figure 1a] decouples the graphene from the Ni electronically and permits the formation of a quasi-free-standing electronic structure including a gapless Dirac cone and almost neutral doping [48]. At the same time, it is responsible for the spinorbit interaction of Rashba type [Figure 1b]. The magnitude of spin-orbit splitting, marked in Figure 1b, remains constant with momentum which is a hallmark of the Rashba effect on massless Dirac fermions [41-43]. Our ab-initio calculations [15] show that this splitting depends strongly on the graphene-Au distance, as Figure 1c shows. The band topology of Figure 1b, in particular the gapless character, is for small distances only maintained when the $\mathrm{Au}$ monolayer atoms are in the hollow site of the graphene (Figure 1a). In Figure 1c, the equilibrium distance is denoted as $3.3 \AA$. This value is also in agreement with previous results for graphene/ $\mathrm{Au}(111)$ [8]. Figure 1c shows the spin-orbit splittings measured in the experiment [regions (1) and (2)] vis-à-vis the calculated distance dependence for the sharp graphene-Au interface.

To support the plausibility of a the giant splitting, which requires a closer distance be- 
tween Au and graphene in equilibrium, a $p(2 \times 2)$ superstructure of Au atoms was investigated in a proof-of-principle calculations [15]. This structure which has a reduced atom density of 0.25 ML lets the Au atoms sink deeper into the graphene and reaches a giant splitting of 60 meV at $2.3 \AA$ equilibrium separation [15]. However, this or a similar superstructure has not been confirmed by STM measurements and remained therefore hypothetic.

In search for a structural origin of the giant spin-orbit splitting we have in the present work performed an extensive STM characterization of Auintercalated graphene/ $\mathrm{Ni}(111)$. In order to corroborate our previous photoemission results[15] we compare the intercalation of graphene grown on $\mathrm{Ni}(111)$ single crystals and on $\mathrm{Ni}(111)$ prepared as a thin film on W(110). Graphene grown on a Ni thin film generally displays higher structural coherence with the substrate as seen from the absence of rotational disorder. The reason is that the Ni film shows no bulk-derived screw-dislocations at the surface and a very weak 1D stripe-like pattern with lateral periodicity of 8.5-11 nm, which stems from the relief of strain induced by W(110). Nevertheless, regarding the types of structures formed by the intercalated $\mathrm{Au}$, we did not observe a qualitative difference between the substrates. In both cases we observe the formation of various intercalated $\mathrm{Au}$ nanocluster arrays which are coherent to and coexist with the conventional monolayer moiré phase identified earlier as $p(\mathbf{9} \times \mathbf{9})[15]$ (Figures 2, 3 and $\mathrm{S} 1$ show $\mathrm{Ni}(111)$ single crystal, for thin film $\mathrm{Ni}(111) / \mathrm{W}(110)$ see Figure S2). We suggest these nanoclusters as the origin of the giant Rashba effect. The enhancement of the Au-induced spin-orbit interaction by an order of magnitude as compared to the one of graphene on the continuous moiré-type $\mathrm{Au}$ monolayer is ascribed to the reduced distance between graphene and $\mathrm{Au}$ nanocluster caused by the attraction of graphene by the open $\mathrm{Ni}$ areas between the Au clusters. Our conclusions are supported by density functional theory (DFT) calculations. We speculate, however, that the rotational disorder may modify the intercalation pathway and favour the Au monolayer responsible for the spin-orbit splitting of $\sim 10 \mathrm{meV}$ observed in our previous publication (Ref. [48]). Another possibility is that the rotational disorder directly reduces the spin-orbit splitting. This is the mechanism we discovered for rotated graphene on $\operatorname{Ir}(111)[29]$. 

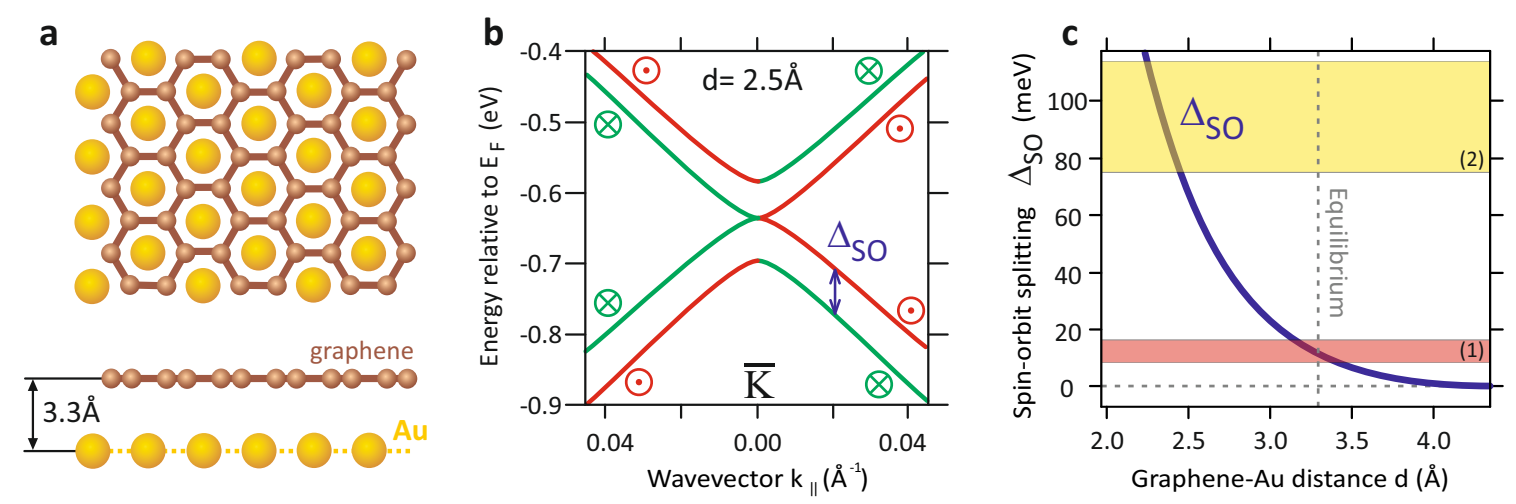

FIG. 1: Rashba-type spin-orbit splitting in graphene calculated within the proximity of a continuous monolayer of Au. (a) Structural model shows mutual arrangement of graphene and Au lattices. The hollow-site configuration (Au atom in the center of graphene hexagon) ensures preservation of the gapless Dirac cone. The equilibrium separation between graphene and $\mathrm{Au}$ is $3.3 \AA$. (b) Dirac cone of graphene pushed close to $\mathrm{Au}$ at non-equilibrium separation of $2.5 \AA$. Spin-orbit splitting $\Delta_{\text {SO }}$ is as large as $70 \mathrm{meV}$. Red and green colors denote directions of spin. (c) Overall dependence of spin-orbit splitting of Dirac cone in graphene on the distance to Au monolayer. In addition, experimental results from Refs. $[15,48]$ are given. The magnitude ranges of $\Delta_{\text {SO }}$ acquired for different wavevectors $\mathbf{k}_{\|}$: (1) for graphene on $\mathrm{Au} / \mathrm{Ni}(111)$ single crystal and (2) for graphene on $\mathrm{Au} / \mathrm{Ni} / \mathrm{W}(110)$. Average values of $\Delta_{\mathrm{SO}}$ are determined as 13 and $96 \mathrm{meV}$, respectively.

\section{RESULTS AND DISCUSSION}

\section{A. Phase I. Intercalated continuous monolayer of Au and its moiré pattern}

We start with the description of the most common phase of intercalated Au: the continuous monolayer phase. It is usually considered that submonolayer amounts of Au intercalated at the graphene-Ni interface form continuous monolayer-thick islands. Since the lattice constant of $\mathrm{Au}$ is by $\sim 10 \%$ larger than that of $\mathrm{Ni}$ and graphene, the formation of a moiré pattern such as $p(9 \times 9)$ is expected.

Figure 2 shows the characterization of graphene grown on a $\mathrm{Ni}(111)$ film and intercalated with 0.5 ML of Au. Figure 2a shows a large scale STM image with multiple islands seen. Figure $2 \mathrm{~b}$ displays a zoom into such islands. One sees that the islands host a periodic hexagonal pattern resembling a moiré effect pattern which is expected to appear due to the lattice mismatch between $\mathrm{Ni}$ substrate and a monolayer of gold. In this way, islands 

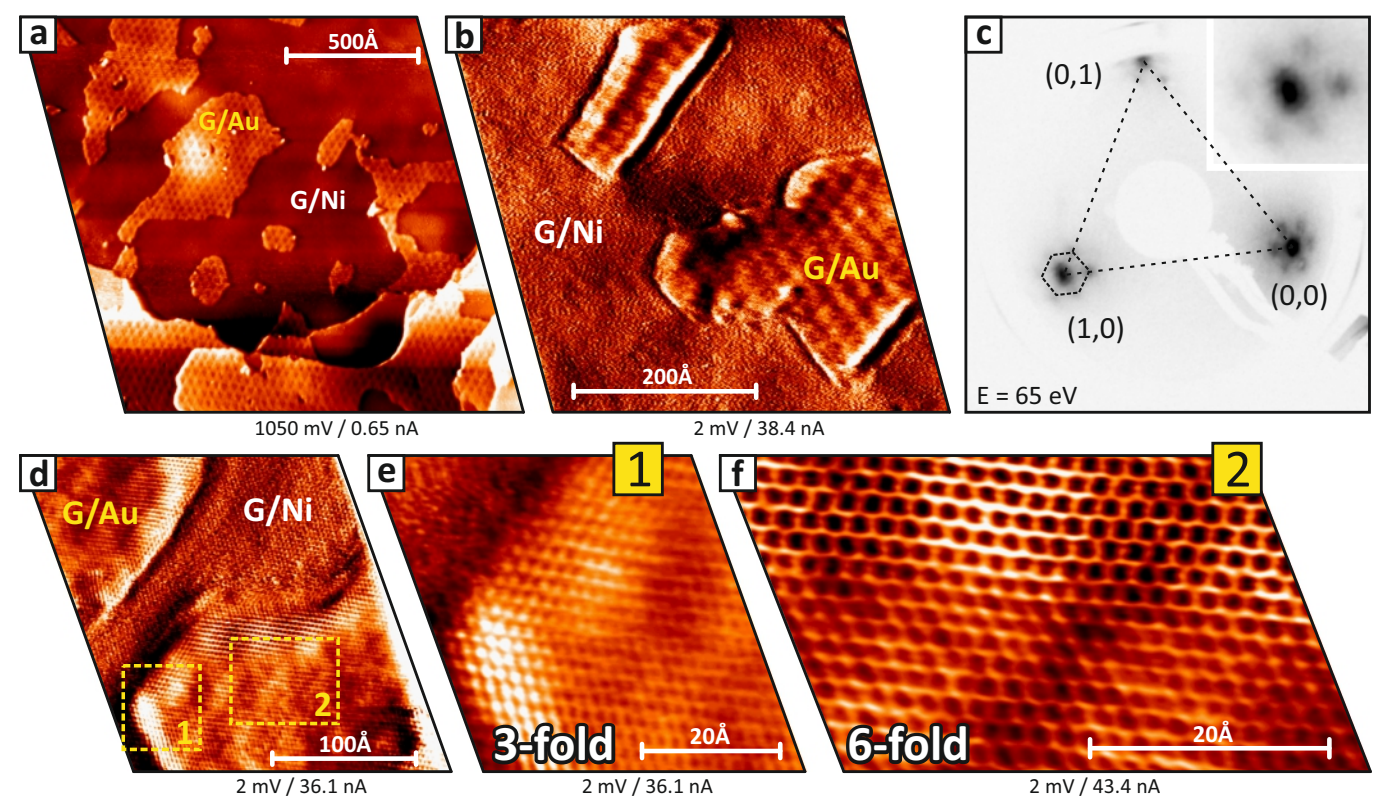

FIG. 2: STM characterization of Au intercalated under graphene on $\mathrm{Ni}(111)$ in the continuous monolayer phase with moiré pattern. Results are obtained for 0.5 ML Au intercalated under graphene on a Ni film atop W(110). (a,b) Large scale images reveal monolayer-thick Au islands (labeled $\mathrm{G} / \mathrm{Au}$ ) of different sizes with a pronounced moiré pattern (periodicty $\sim 25 \AA$ ). Areas between islands (labeled $\mathrm{G} / \mathrm{Ni}$ ) are the areas of bare graphene/Ni. They do not show moiré-like superstructures. (c) LEED pattern of graphene/Ni(111) intercalated with nearly full Au monolayer reveals a Au-induced moiré pattern through superstructural constellations around primary spots. The pattern is determined as $(9.7 \times 9.7)$ superstructure and identified as misfit dislocation loop structure induced by Au-Ni surface alloying [see Figure $3 \mathrm{f}$ and discussion there]. (d,e,f) Zoom of two intercalated $\mathrm{Au}$ islands with atomic resolution. Enhanced zooms on island rim [area 1] and island interior [area 2] are shown in (e) and (f), respectively. In contrast to the rims of $\mathrm{Au}$ islands, graphene above the inner areas of islands demonstrates predominantly 6 -fold symmetric honeycomb lattice which manifests preservation of sublattice symmetry.

were identified as patches of a Au monolayer. Evaluation of STM data and LEED pattern acquired from the sample of Figure 2c allows for a rough identification of the observed moiré pattern as $\sim p(9 \times 9)$ with superstructural periodicity of $\sim 25 \AA$ (along principle directions of hexagonal moiré superlattice).

Furthermore, we emphasize that $\mathrm{Au}$ intercalated under graphene on $\mathrm{Ni}(111)$ forms a single monolayer thick film at the interface. The topography signal alone cannot provide 
such evidence and cannot measure the height of intercalated Au islands properly because of the very strong electronic contrast between intercalated and non-intercalated graphene areas [38]. This is ascribed to different density of states in the vicinity of the Fermi level of graphene bonded to $\mathrm{Ni}$ and graphene decoupled from $\mathrm{Ni}$ by $\mathrm{Au}$. Indeed, by selection of the specific bias voltage at the tip one can achieve either strong contrast of Au islands $(\sim 1.5$ $\AA$, like in Figure 2a measured with $\mathrm{V}_{\text {bias }}=1 \mathrm{~V}$ ) or reduce the contrast nearly to zero [like in Figure $2 \mathrm{~b}$ measured with $\mathrm{V}_{\text {bias }}=2 \mathrm{mV}$ ]. By careful selection of the tunneling parameters, we could see through graphene and resolve the inner structure of the $\sim p(9 \times 9)$ moiré in greater detail. This result is reported in Figure $3 \mathrm{f}$ (the data was acquired for a single crystal Ni substrate). One sees that the apparent $\sim p(9 \times 9)$ superstructure occurs as an array of triangles which, according to an earlier study of a single monolayer of $\mathrm{Au}$ on top of $\mathrm{Ni}(111)$, are formed by gold atoms dipping in the triangular misfit dislocation loops occurring in the Ni uppermost layer [51]. Formation of such dislocation loops can be explained in terms of reconstruction of the topmost $\mathrm{Ni}$ layer which is energetically favorable due to interaction with the gold monolayer. Note that the graphene matches perfectly the $\mathrm{Ni}(111)$ so that the superstructure of the Au can result either from the graphene-Au or from the Au-Ni interface. The inset in Figure 3f shows a zoom of this triangular pattern with atomically resolved graphene overlayer. The observation of a nearly perfect honeycomb lattice superimposed on the triangles shows that $\mathrm{Au}$ is indeed intercalated under the graphene and that structural integrity and quality of graphene lattice are preserved upon intercalation.

These observations prove that: (i) intercalated Au indeed forms a $1 \mathrm{ML}$ thick film, (ii) the moiré structure seen in graphene by STM originates from Au on Ni and to lesser extent from the graphene-Au interface (iii) the moiré pattern is in reality not $p(9 \times 9)$ but a peculiar $(9.7 \times 9.7)$ structure occurring due to misfit dislocation loops in the $\mathrm{Ni}(111)$ substrate caused by $\mathrm{Ni}-\mathrm{Au}$ surface alloying [51].

Our results demonstrate that a truly continuous monolayer of Au with a conventional primitive $p(9 \times 9)$ moiré structure does not realize for the intercalated Au interlayer. According to Ref. [51], the moiré phase of $1 \mathrm{ML} \mathrm{Au} / \mathrm{Ni}(111)$ is not compatible with annealing, which, in our case, is required for the activation of $\mathrm{Au}$ intercalation. A truly continuous Au layer may exist on $\mathrm{Ni}(111)$ but not for temperatures above room temperature. After any moderate annealing it switches toward a $(9.7 \times 9.7)$ surface alloy [51]. As we see, the presence of graphene overlayer does not change this behavior. 
Let us now focus on the structure of graphene hosted on the continuous Au monolayer with $(9.7 \times 9.7)$ moiré structure. Figure $2 \mathrm{~d}$ displays an enlarged atomically resolved image of graphene atop a $\mathrm{Au}$ island. Parts e and $\mathrm{f}$ of Figure 2 display zooms of the two areas denoted in Figure 2d with yellow dashed rectangles. Area 1 [Figure 2e] shows the graphene lattice close to the edge of the $\mathrm{Au}$ island while in area 2 the atomically resolved image was taken from the inner part of the $\mathrm{Au}$ island. It is worthwhile noting that the symmetry of the graphene lattice is different in areas 1 and 2. Within the interior of the Au island, the graphene predominantly reveals a 6-fold symmetry (manifested by the observation of a nearly perfect honeycomb structure) but at the rims of the Au islands the symmetry is remarkably 3 -fold. This means that in graphene on the continuous Au layer the sublattice symmetry is not broken. This is consistent with its gapless Dirac cone [10]. The disturbed graphene symmetry observed in our earlier work [10] only appears at the rims of Au islands (or completely for small islands) due to a transition toward the top-fcc arrangement of graphene/ $\mathrm{Ni}(111)$ with a naturally extreme sublattice asymmetry [23].

Furthermore, co-observation and analysis of the $(9.7 \times 9.7)$ moiré pattern on the one hand and the atomically resolved graphene lattice on the other hand [Figures $2 \mathrm{~d}$ and $3 \mathrm{f}$ ] lead to the conclusion that the graphene lattice is perfectly aligned to the lattice of the intercalated $\mathrm{Au}$ monolayer. This finding contrasts earlier reports claiming that intercalation of $\mathrm{Au}$ at low concentrations only takes place for rotationally displaced graphene variants [52].

\section{B. Phase II. Intercalated superlattices of Au clusters}

Along with the continuous monolayer phase [(9.7×9.7) moiré] we systematically observe in our samples two other phases of intercalated Au occurring as periodic arrays of Au nanoclusters. These arrays vary in their lateral dimensions but occur in every sample prepared where they co-exist with the continuous monolayer phase. We observe them for different concentrations of $\mathrm{Au}$ and for both types of the $\mathrm{Ni}(111)$ substrate (single crystal and Ni film on top of tungsten). The lateral dimensions of these arrays vary from 20 to $200 \mathrm{~nm}$ while their lateral separation from each other is rather random but not exceeding $400 \mathrm{~nm}$. It must be emphasized that the occurrence of the Au cluster arrays on $\mathrm{Ni}(111)$ is a unique feature emerging only in the presence of the graphene overlayer. Intercalation of gold under graphene in form of few-atom clusters was previously reported for graphene on $\mathrm{SiC}(0001)$, 
however the arrangement of intercalated particles was rather irregular. [53] Authors estimate that $\mathrm{Au}$ clusters form a disordered $(9 \times 9)$ reconstruction with respect to the graphene lattice with an average distance between the clusters of $2.2 \mathrm{~nm}$. [53]

The intercalated clusters of gold on $\mathrm{Ni}(111)$ are displayed in Figure 3. Figure 3a shows a large scale STM image. One sees several structural phases of Au under graphene, which are denoted in the figure with corresponding labels. The first phase is an array of large $(\sim 1$ $\mathrm{nm})$ nanoclusters with periodicity of $\sim 25 \AA$ and $z$-corrugation of $\sim 1.5 \AA$ [see height profile $\mathrm{S}_{1}$ in Figure 3b]. The periodicity of $\sim 25 \AA$ is essentially the same as of the moire phase $(9.7 \times 9.7)$ of the continuous Au monolayer [Figure 2]. This demonstrates that the occurrence of the periodic nanocluster array is closely related to the mechanism of the $(9.7 \times 9.7)$ moiré formation in the continuous monolayer of $\mathrm{Au}$ and has essentially the same origin which is the large $(\sim 16 \%)$ lattice mismatch between $\mathrm{Au}$ and $\mathrm{Ni}$.

Also another type of nanocluster array is seen. The superlattice of this array is rotated by $30^{\circ}$ relative to the $(9.7 \times 9.7)$ structure and has a smaller periodicity of $\sim 17 \AA$ and smaller $z$-corrugation of $\sim 0.5 \AA$ [see height profile $\mathrm{S}_{2}$ in Figure $3 \mathrm{~b}$ ]. In addition, the nanoclusters have smaller size $(\sim 0.5 \mathrm{~nm})$. At first sight, this cluster phase can be regarded as $\sim(3 \sqrt{3} \times$ $3 \sqrt{3}$ ) $\mathrm{R}^{\circ}$ (a more precise analysis given below will identify it as $(3.2 \sqrt{3} \times 3.2 \sqrt{3})$ R30 based on its lateral coherence to the $(9.7 \times 9.7)$ superstructure). Both cluster phases co-exist with the $(9.7 \times 9.7)$ phase of the continuous Au monolayer which is labeled correspondingly throughout the STM images in Figure 3. The $(9.7 \times 9.7)$ moiré on the continuous monolayer area is not clearly resolved in Figure 3a because of different optimal tunneling parameters for the observation of the cluster and moiré phases. It is much better seen in Figures 3d and $3 f$ which have been acquired with different bias voltage allowing to see through the graphene: there appear characteristic triangles forming the moiré pattern of the $(9.7 \times 9.7)$ monolayer phase.

We mentioned above that the $(9.7 \times 9.7)$ pattern occurs due to misfit dislocation loops in the Ni substrate caused by partial Au-Ni surface alloying [51]. The simultaneous appearance of the monolayer phase with $(9.7 \times 9.7)$ moiré and the $(9.7 \times 9.7)$ and $\sim(3 \sqrt{3} \times 3 \sqrt{3})$ R30 cluster phases in the same STM images in Figure 3 indicate that the cluster phase is indeed composed of individual nanoclusters and is not an instrumental artefact during the imaging of conventional moiré superstructures.

We conclude that structurally these individual nanoclusters are small patches of a $\mathrm{Au}$ 

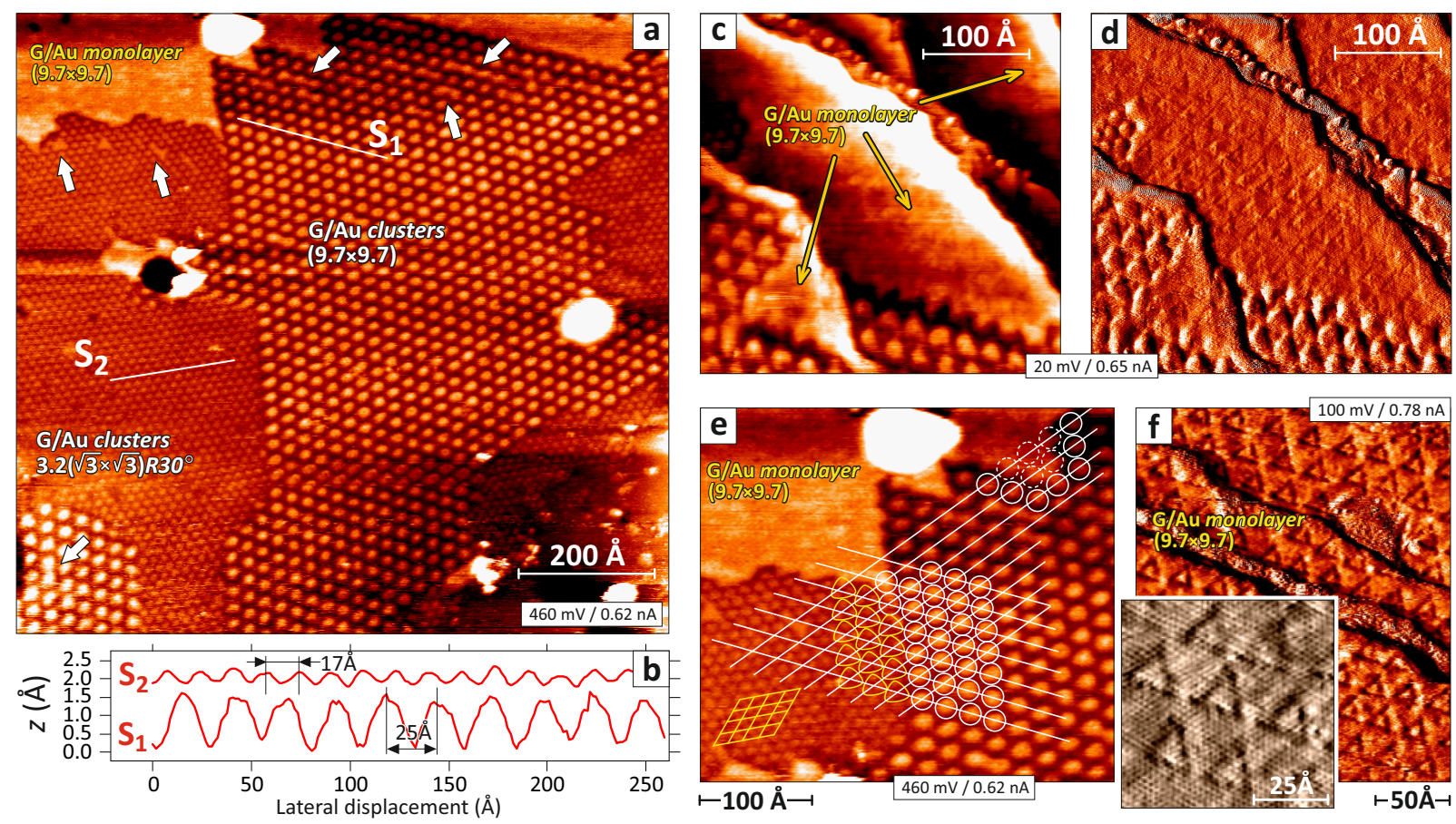

FIG. 3: Observation of arrays of intercalated clusters. Experiments were performed on Ni single crystals and nominally 0.5 ML Au. (a) Large scale STM image showing co-existence of a $(9.7 \times 9.7)$ moiré pattern of the continuous monolayer phase, a $(9.7 \times 9.7)$ array of large clusters and an array of small clusters identified as $(3.2 \sqrt{3} \times 3.2 \sqrt{3})$ R30 . White arrows denote sites where coalescence (or dealloying) of individual clusters toward continuous monolayer phase takes place. In (b) one can see height profiles along the lines $\mathrm{S}_{1}$ and $\mathrm{S}_{2}$. (c) and (d) show zoom of cluster array edge as topography and tunneling current channels, respectively. These images also show co-existence of multiple structural phases. A continuous Au monolayer phase giving rise to the $(9.7 \times 9.7)$ moiré pattern is identified as misfit dislocation loop structure caused by surface alloying between Au and $\mathrm{Ni}$ [51] by its characteristic pattern composed of triangles which are seen by STM through graphene. This pattern is better observed in image (d) which represents modulation of tunneling current with reduced feedback sensitivity and hence is more sensitive to fine structural features; (e) Graphical plot evidencing lateral coherence between all three types of intercalated Au structures (continuous monolayer with moiré, $(9.7 \times 9.7)$ clusters and $(3.2 \sqrt{3} \times 3.2 \sqrt{3})$ R30 clusters). (f) Observation of a $(9.7 \times 9.7)$ misfit dislocation loop structure through graphene at enhanced resolution achieved by proper optimization of tunneling parameters. Zoom shown in the inset reports simultaneous observation of triangular pattern of intercalated $\mathrm{Au}$ and graphene mesh on top with atomic resolution. 
monolayer periodically arranged under the graphene. This claim is supported by several experimental evidences. The first proof, as mentioned above, arises from the co-observation of the cluster phases and the $(9.7 \times 9.7)$ continuous $\mathrm{Au}$ monolayer. The second argument is that the bigger clusters reveal the same absolute $z$-height in the STM as the continuous $(9.7 \times 9.7)$ phase and their lateral contrast (difference of $z$-signals from clusters and areas between clusters) shows the same behavior with tunneling bias as large islands of the continuous monolayer phase do [Figure 3]. This means that the nanoclusters seen by STM are similar electronically and structurally to large Au islands. As a third argument serves their coalescence into a continuous $\mathrm{Au}$ film, observed locally for both $(9.7 \times 9.7)$ and $\sim(3 \sqrt{3} \times$ $3 \sqrt{3})$ R30 nanocluster arrays. Such coalescent clusters are pointed out in Figure 3a with white arrows. Coalescence effects of similar occurrence have been observed by STM for Si nanoclusters intercalated under graphene on $\mathrm{Ru}$ [54].

The zoom in parts c and d of Figure 3 (topography and tunneling current channels, respectively) shows the transition from the $(9.7 \times 9.7)$ phase of the continuous $\mathrm{Au}$ monolayer to the $(9.7 \times 9.7)$ cluster phase. Here, one clearly sees the equality of $z$-signals from clusters and from continuous Au islands. The rim of the continuous Au layer has a sawtooth profile which follows the moiré pattern. Beyond this sawtooth edge, the Au clusters occur individually and reside within a $(9.7 \times 9.7)$ network of moiré cells. The significantly different shape of the clusters is a fourth evidence that they are individual nanoclusters and not a kind of moiré imaging effect.

In view of the apparent structural correlations between cluster arrays and $(9.7 \times 9.7)$ moiré of continuous monolayer, we have performed a coherence analysis of large scale STM data shown in Figure 3a. The coherence plot is presented in Figure 3e. The STM data is superimposed on a $(9.7 \times 9.7)$ superstructural grid and one sees that large clusters are fully coherent to the $(9.7 \times 9.7)$ moiré of the continuous $\mathrm{Au}$ monolayer: they precisely arrange in the same moiré matrix. Furthermore, small clusters of the $\sim(3 \sqrt{3} \times 3 \sqrt{3})$ R30 phase are also in registry to $(9.7 \times 9.7)$ - if one joins every three of them, one gets a $(9.7 \times 9.7)$ matrix which is fully coherent to the arrangement of large clusters and to the moiré pattern of the continuous monolayer phase. This, in turn, means that all observed cluster arrays originate from the lattice mismatch between $\mathrm{Au}$ and $\mathrm{Ni}$ and emerge as incomplete moiré pattern due to lower local concentration of $\mathrm{Au}$. Such requirement of global coherence allows to identify the superstructure of small clusters more precisely as $(3.2 \sqrt{3} \times 3.2 \sqrt{3}) \mathrm{R} 30^{\circ}$. 
We estimate that the ratio between the areas occupied with clusters and the continuous monolayer phase varies in the range $10-30 \%$, depending on sample preparation. This is a conservative estimate extracted from STM characterizations of 3-4 macroscopic areas of each sample prepared. We emphasize that this might be not representative for the whole sample surface due to local nature of scanning probing techniques. In Ref. [15] we underestimated the extent of $\mathrm{Au}$ clusters because they lead to the same superstructure as the full $\mathrm{Au}$ monolayer. In some STM measurements we have indeed observed more than $50 \%$ of Auintercalated surface area, Supplementary Fig. S1 shows an example of a wide area $\left(\sim 300 \times 300 \mathrm{~nm}^{2}\right)$ STM image with a $\sim \mathbf{5 0 \%}$ cluster coverage.

We believe that the observed arrays of intercalated Au nanoclusters are likely the source of the experimentally observed giant spin-orbit splitting of the Dirac cone of Figure 1. In the following we report theoretical data supporting this claim.

C. Density functional theory study of Au-intercalated graphene

In order to establish the role of Au clusters for the spin-orbit splitting in graphene scanning tunneling spectroscopy (STS) is not directly applicable. Neither direct STS measurements, nor quantum interference patterns (QIP) born by scattering of quasiparticles on structural defects can provide consistent information about the spin structure of $2 \mathrm{D}$ electronic states $[55,56]$ because of the blocking of intraband scattering between the states of opposite spin [57]. Graphene flakes on $\mathrm{Au}(111)$ have recently been used to probe the $\mathrm{Au}$ surface state and indirectly a possible Rashba splitting of the graphene [58]. This method is not applicable to the present system since it requires the presence of the $\mathrm{Au}(111)$ surface state which does not develop for $\mathrm{Au}$ on $\mathrm{Ni}(111)$. Due to this, and taking into account that alternative local spectroscopic techniques like nano-ARPES are not yet affordable with spin-resolution, we see theoretical simulation of graphene intercalated with Au clusters as the only available method of study.

To understand the effect of intercalated clusters on electronic and magnetic properties of graphene we have performed a series of theoretical simulations. 
In our earlier work in Ref. [15] we have studied the effect of a $(2 \times 2)$ superstructure of $\mathrm{Au}$ adatoms on the spin-orbit splitting in graphene. It was demonstrated by ab initio theory that by diluting a $\mathrm{Au}$ monolayer in the $0.25 \mathrm{ML}(2 \times 2)$ structure, the equilibrium graphene- $\mathrm{Au}$ distance strongly reduces to $2.3 \AA$. This, in turn, induces a giant $(\sim 90 \pm 15 \mathrm{meV})$ spin-orbit splitting in the graphene Dirac cone, consistent with the experimentally observable value [Figure 1c].

From this starting point we investigate whether small Au nanoclusters intercalated under graphene on $\mathrm{Ni}(111)$ could play a role similar to that of $\mathrm{Au}$ adatoms mentioned above. For a rough estimate of the induced spin-orbit effect the dependence of spin-splitting on the graphene-Au distance of the continuous Au monolayer [Figure 1c] can be used.

To search for an enhanced splitting and to understand the possible physical mechanism, we performed structural calculations for various models of graphene in contact to small $\mathrm{Au}$ clusters with and without inclusion of the $\mathrm{Ni}(111)$ substrate. Due to constraints of computational resources we investigated only small $\mathrm{Au}$ units: the single $\mathrm{Au}$ adatom $\left(\mathrm{Au}_{1}\right)$ and a cluster composed of three $\mathrm{Au}$ atoms $\left(\mathrm{Au}_{3}\right.$ molecule).

In our setup, $\mathrm{Au}_{1}$ or $\mathrm{Au}_{3}$ molecules were placed into a slab composed of a sheet of graphene and monolayer of Ni representing the $\mathrm{Ni}(111)$ substrate. Slab dimensions were selected as $(6 \times 6)$ and $(10 \times 10)$ graphene hexagons for $\mathrm{Au}_{1}$ and $\mathrm{Au}_{3}$ molecules, respectively. The lateral arrangements of atoms in these structural models are shown in Figure 4a,b. These dimensions are in line with experimental data as they correlate very well with the periodicities of observed $(3.2 \sqrt{3} \times 3.2 \sqrt{3}) \mathrm{R}^{\circ}(17 \AA)$ and $(9.7 \times 9.7)(25 \AA)$ superstructures. The structures with implanted Au clusters were allowed to relax from two diametrical starting points. The first configuration comprised a flat graphene supercell initially placed about $3 \AA$ above the Au cluster, while the second one was artificially deformed around the cluster to create a buckled graphene structure and at the same time reduce the initial distance between $\mathrm{C}$ atoms and the $\mathrm{Ni}(111)$ substrate. Subsequently, the structure of graphene was allowed to relax. In the case of the (6x6) supercell, the most stable configuration is depicted in Figure $4 \mathrm{c}$, and corresponds to the initially flat structure. The initially bent structure relaxes to a buckled configuration (not shown here) with higher corrugation, which is slightly less stable than the flat graphene ( $<8 \mathrm{meV}$ per $\mathrm{C}$ atom) due to the reduced supercell size for stress discharge. This is consistent with the enhanced stability of both corrugated structures (see Figure 4e,f), as compared to the quasi-free-standing flat graphene, for the structural 

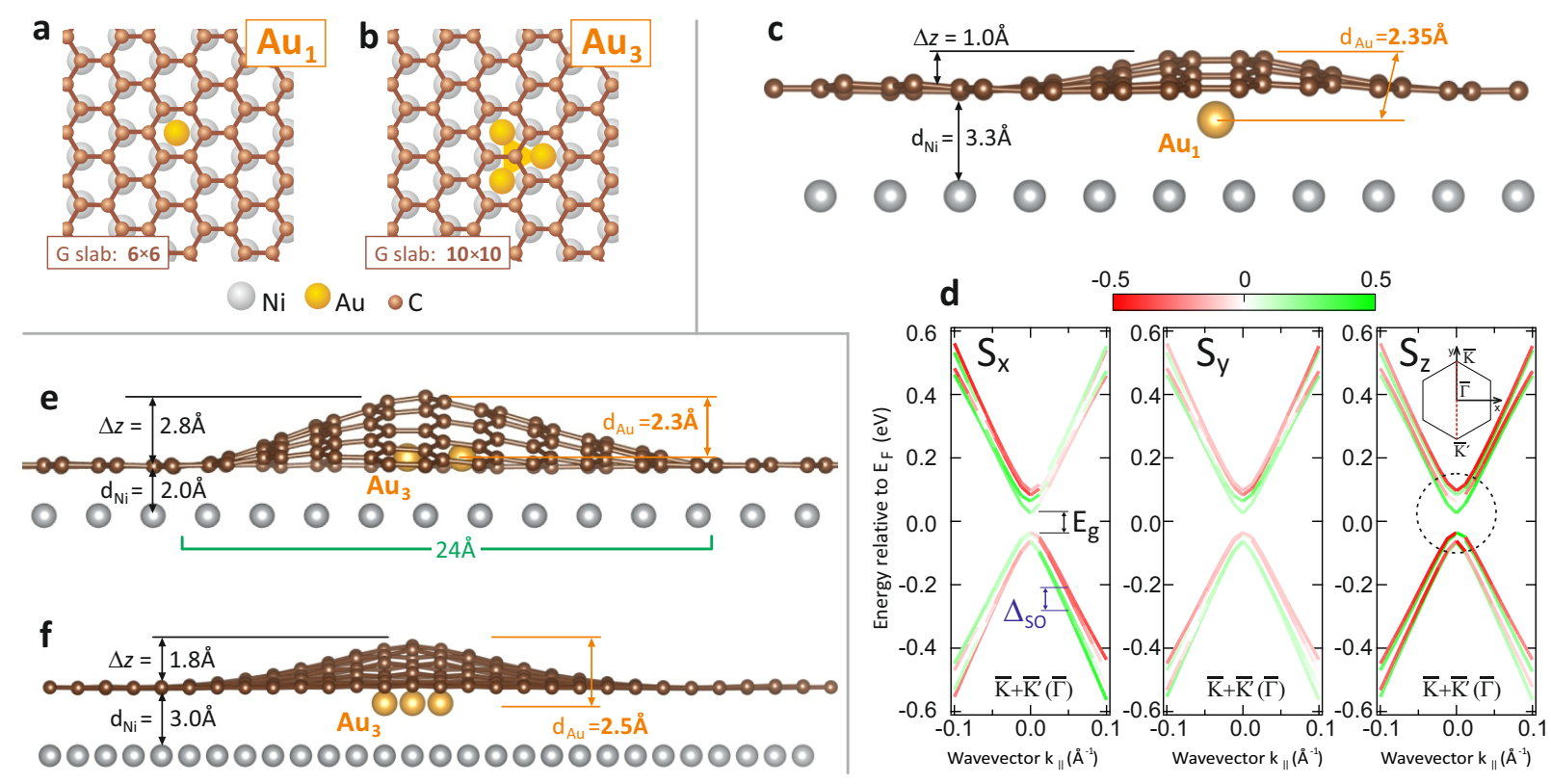

FIG. 4: DFT study of graphene intercalated with arrays of small Au clusters. (a,b) Structural models showing the lateral arrangement in the initial setup of $\mathrm{Au}_{1}$ (single atom) and $\mathrm{Au}_{3}$ atomic clusters within the graphene/Ni slab, respectively. Slab dimensions are $(6 \times 6)$ for calculation of $\mathrm{Au}_{1}$ and $(10 \times 10)$ for $\mathrm{Au}_{3}$. (c) Relaxed atomic structure acquired for $\mathrm{Au}_{1}$ in $(6 \times 6)$ graphene/Ni matrix. The large graphene-Ni distance $\left(d_{N i}=3.3 \AA\right)$ indicates that graphene becomes quasi-free-standing. The small separation between graphene and $\mathrm{Au}\left(d_{A u}=2.35 \AA\right)$ suggests strong local spin-orbit effects in the graphene. (d) Fully relativistic band structure calculation for the system from (c) (with Ni substrate excluded) revealing large Rashba-type spin-orbit splitting of Dirac cone (projections $\mathrm{S}_{x}$ and $\mathrm{S}_{y}$ ) but also development of a substantial out-of-plane spin component (projections $\mathrm{S}_{z}$ ) within the gap of Dirac cone (dashed circle) caused by structural buckling of graphene. (e,f) Relaxed structures obtained for $\mathrm{Au}_{3}$ in $(10 \times 10)$ graphene/Ni matrix. The two structures have almost equal energies but very different properties. Structure shown in (e) corresponds to configuration in which graphene is strongly bonded to $\mathrm{Ni}\left(d_{N i}=2.0 \AA\right)$. As a result, a giant structural corrugation $\left(\Delta_{z}=2.8\right.$ $\AA)$ with enhanced proximity to $\mathrm{Au}\left(d_{A u}=2.3 \AA\right)$ evolves. The second structure shown in (f) reveals quasi-free-standing graphene weakly bonded to $\mathrm{Ni}\left(d_{N i}=3.0 \AA\right)$ with smaller corrugation $\left(\Delta_{z}=1.8\right.$ $\AA)$ and smaller distance to Au cluster $\left(d_{A u}=2.5 \AA\right)$ which, however, according to Figure 1c, is enough to induce in graphene a giant spin-orbit splitting of about $70 \mathrm{meV}$. The structure shown in (e) is metastable and easily switches toward configuration of quasi-free-standing graphene (f) by thermal energies. 
calculations of the $(10 \times 10)$ supercell.

The results of our simulation confirm the initial hypothesis that interaction between graphene and the $\mathrm{Ni}$ substrate plays a crucial role for enhancing the proximity of graphene to the intercalated Au cluster. Most specifically, the calculations reveal an interplay between two competing mechanisms, namely, proximity with the $\mathrm{Ni}(111)$ substrate that drives the system toward stability and bending of the graphene sheet, which increases the total energy of the system.

Let us discuss the results of the simulations in detail. We start with description of relaxed graphene obtained for the single $\mathrm{Au}$ atom $\left(\mathrm{Au}_{1}\right)$ in the $(6 \times 6)$ graphene/Ni slab. This is a showcase model with the help of which we learn how interaction with $\mathrm{Au}$ and the related corrugation affect the graphene properties. Figure 4c displays the final structure obtained by the simulations. One sees that graphene buckles above the $\mathrm{Au}$ atoms with a corrugation of $\Delta z \sim 1 \AA$. At the same time the minimal separation between graphene and the atomic layer of $\mathrm{Ni}$ becomes $d_{N i}=3.3 \AA$ which is substantially larger than the equilibrium distance between graphene and $\mathrm{Ni}(111)$ in the non-intercalated system $\left(d_{N i}=2.05 \AA\right)$, obtained by DFT calculations in Refs. [8, 9]. Those papers established the criterion of weakly bonded quasi-free-standing graphene on metals, i.e., a distance between graphene and its substrate larger than $3 \AA$. From this one clearly sees that even small amounts of intercalated $\mathrm{Au}$ forming superstructures or sieves do effectively decouple graphene from the $\mathrm{Ni}$, reduce the effect of $\mathrm{Ni}$ on the sublattice asymmetry and/or doping, i.e., render it quasi-free-standing.

Most important, however, is the minimal distance between $\mathrm{Au}$ atom and graphene which is determined from the model in Figure $4 \mathrm{c}$ as $\mathrm{d}_{A u}=2.35 \AA$. According to the calculation for a continuous Au layer reported in Figure 1c at such small separation one can expect a spin-orbit splitting in the graphene as large as $100 \mathrm{meV}$. In this context, it is particularly intriguing to estimate the strength of spin-orbit coupling induced in the band structure of graphene not by the flat $\mathrm{Au}$ monolayer but within a more complex model case of $\mathrm{Au}_{1}$ trapped at the graphene/Ni interface. Another question concerns the effect of graphene buckling around the Au cluster on the bands of graphene.

For relativistic band structure calculations we have used the relaxed model shown in Figure 4c but with the Ni substrate removed. This was required to make the computations affordable but is also physically justified due to very large separation between graphene and $\mathrm{Ni}\left(\mathrm{d}_{N i}>3 \AA\right)$. The obtained band structure fully accounting for spin-orbit interactions 
is shown in Figure 4d. Because of the rather complex spin-texture caused by 3D spatial buckling of graphene around $\mathrm{Au}_{1}$, the results are presented in three panels for the three spatial components of spin $\left(\mathrm{S}_{x}, \mathrm{~S}_{y}\right.$ and $\left.\mathrm{S}_{z}\right)$. $\mathrm{S}_{z}$ denotes the spin component perpendicular to the basis plane of flat graphene, while $\mathrm{S}_{x}$ and $\mathrm{S}_{y}$ are spin projections onto in-plane axes. Spins of opposite sign are denoted by green and red colors. The results represent superposition of bands from $\overline{\mathrm{K}}$ and $\overline{\mathrm{K}^{\prime}}$ points which are backfolded together to $\bar{\Gamma}$ because of the $(6 \times 6)$ dimensions of the slab. Dirac cones shown in panels $\mathrm{S}_{x}, \mathrm{~S}_{y}$ reveal a giant Rashba-type spinorbit splitting of $\sim 60 \mathrm{meV}$. Interestingly, the out-of plane spin-components in panel $\mathrm{S}_{z}$ are non-zero in the vicinity of the gap at the Dirac point (emphasized by dashed circle in panel $\mathrm{S}_{z}$ ). This indicates precession of spins related to the buckling of the graphene layer around the Au cluster. This can be ascribed to sublattice asymmetry caused by the buckling which, in combination with spin-orbit interactions enhanced by the intercalated Au particle, causes out-of-plane reorientation of spins in the gap and formation of spin hedgehogs [49, 50]. This, in turn, leads to the important conclusion that nanoarrays of intercalated high- $Z$ materials may effectively be employed for the creation of 3D spin textures in graphene.

Another remarkable effect of the graphene buckling is the band gap at the Dirac point seen in the band structures shown in Figure $4 \mathrm{~d}$. The gap $\mathrm{E}_{g}$ is as large as $60 \mathrm{meV}$ and can either be caused by local sublattice asymmetry or by the electronic confinement in the buckled graphene dome. To figure out which effect plays the major role in the gap formation we have calculated the band structure for the same corrugated graphene sheet but with gold atoms removed. The obtained value of the band gap width in this case is 54 meV. Such a small difference in $\mathrm{E}_{g}$ after removing the $\mathrm{Au}$ atoms indicates that the buckling is a determinative factor for the band gap. Therefore, the graphene buckled above the Au nanocluster cannot be fully equivalent to ideal graphene but possesses a relatively small gap.

Furthermore, we also made an effort to study larger intercalated clusters, which approach the real system better than $\mathrm{Au}_{1}$. The $\mathrm{Au}_{3}$ cluster has been placed in the $(10 \times 10)$ graphene/Ni slab [Figure 4b]. Due to computational limitations only structural properties of this system have been studied. Results are reported in Figures 4e and 4f. In contrast to the case of the single atom $\mathrm{Au}_{1}$, we found two structural configurations with almost equal energy. One configuration, depicted in Figure 4e, corresponds to graphene strongly bonded to the Ni substrate with minimal distance between graphene and Ni layer $\mathrm{d}_{N i}=2.0 \AA$. As a result, a large corrugation $\Delta \mathrm{z} \sim 2.3 \AA$ over the $\mathrm{Au}_{3}$ cluster occurs. Although spin-orbit effects 
induced in such graphene extrinsically must be substantial because the minimal distance between graphene and $\mathrm{Au}$ is small $\left(\mathrm{d}_{A u}=2.3 \AA\right)$, the Dirac cone of graphene must be affected and energetically shifted due to strong interaction with the Ni layer [23]. The band structure corresponding to this structural configuration will not align with the experimental results from Refs. [15, 48]. Its realization is also improbable because it is metastable. Its energy is just slightly higher ( $7 \mathrm{meV}$ per $\mathrm{C}$ atom difference) than the energy of the other configuration in which the graphene is weakly bonded to the Ni. The energy difference between configurations is on the scale of the thermal energy, hence the metastable configuration should evolve toward the more stable structure.

This more favorable structure with weaker bonding between graphene and $\mathrm{Ni}$ is shown in Figure 4f. One sees that the separation between graphene and $\mathrm{Ni}$ is now enlarged $\left(\mathrm{d}_{N i}=3.0\right.$ $\AA$ ) which allows to consider graphene as quasi-free-standing $[8,9]$ with minimal effect of the Ni substrate on the intact Dirac bands. Correspondingly, the magnitude of corrugation over $\mathrm{Au}_{3}$ clusters in the weakly bonded configuration is reduced $(\Delta \mathrm{z} \sim 1.8 \AA)$ and the minimal separation between graphene and $A u$ atoms is enlarged $\left(\mathrm{d}_{A u}=2.5 \AA\right)$. In spite of this, the spin-orbit effect induced in graphene at $\mathrm{d}_{A u}=2.5 \AA$ should still be strong. According to estimates for the proximity of a continuous $\mathrm{Au}$ monolayer (Figure 1), one can expect the spin-orbit splitting to reach $\Delta_{\mathrm{SO}} \sim 70 \mathrm{meV}$ which agrees very well with experimental values [Figure 1]. In addition, the magnitude of the graphene corrugation obtained computationally for the weakly bonded configuration $(\Delta \mathrm{z} \sim 1.8 \AA)$ agrees very well with the value measured in the STM experiment $(\Delta \mathrm{z} \sim 1.5 \AA)$ [Figure 3b].

We conclude that structures very similar to that obtained for $\mathrm{Au}_{1}$ [Figure 4c] and $\mathrm{Au}_{3}$ [Figure 4f] are realized upon intercalation of graphene/ $\mathrm{Ni}(111)$ with $\mathrm{Au}$ and give rise to intact Dirac cones with giant Rashba-type splitting of up to $\Delta_{\mathrm{SO}} \sim 100 \mathrm{meV}$. As mentioned above, the analysis of a large amount of STM data shows that not more than $30 \%$ of the Au-intercalated area of the graphene-Ni interface hosts such arrays of Au clusters while the rest is occupied with the continuous monolayer phase $(9.7 \times 9.7)$. This, in turn, means that giant spin-splitting of the Dirac cone observed in the experiment [15] stems from only part of the spectral weight while the rest of the photoemission signal bears smaller magnitudes of the splitting. This is in line with our photoemission data (see Figure 1 in Ref. [15]) in which the spin-resolved photoemission peaks reveal asymmetric shapes. This was solved in Ref. [15] by fits of the peak shape with small and large magnitudes of the spin splitting. 
Finally, we would like to remark that an enhancement of interaction between graphene and $\mathrm{Au}$ due to Ni cannot be excluded also for the continuous monolayer phase with $(9.7 \times 9.7)$ moiré [Figure 3f]. The misfit dislocation loop structure [Figure 3f] can be considered a Au$\mathrm{Ni}$ surface alloy [51] with individual Ni atoms pushed out of the Ni substrate and floating among $\mathrm{Au}$ at the surface seen in Figure 3f. These Ni atoms may interact with the graphene and pull it towards the substrate hence decreasing the separation (and increasing the spinorbit interaction) between the Au layer and graphene. Understanding of such mechanism requires further investigations. Its computational investigation is presently restricted by the structural complexity of the misfit dislocation loop pattern.

\section{SUMMARY AND CONCLUSIONS}

In the present work we have investigated the question why does the intercalation of $\mathrm{Au}$ under graphene on Ni succeed in causing a Rashba effect of giant magnitude.

Our central result is that $\mathrm{Au}$ forms previously undetected periodic nanoclusters under the graphene. Therefore the graphene and the $\mathrm{Au}$ are not as much decoupled as it could be expected from the intercalation of a flat $\mathrm{Au}$ monolayer. Instead, the graphene remains pinned to the Ni substrate which reduces the distance between graphene and Au sufficiently to enable a graphene-Au hybridization which imposes a strong spin-orbit interaction on the graphene. This conclusion is based on several findings from our structural investigation by STM and DFT simulations. These were:

(i) The discovery of two principally different modes of $\mathrm{Au}$ intercalation under graphene on $\mathrm{Ni}(111)$. In the first regime, a continuous $\mathrm{Au}$ monolayer with $(9.7 \times 9.7)$ moiré pattern is formed. Our STM measurements reveal that this pattern is composed of ordered triangles emerging due to surface alloying between $\mathrm{Au}$ and $\mathrm{Ni}(111)$ which allows us to identify this phase as a misfit dislocation loop structure of the $\mathrm{Au}$ interlayer. In this investigation we remain largely in line with previous literature studies of $\mathrm{Au}$ on pure $\mathrm{Ni}(111)$ which is a famous model system for surface alloying effects.

(ii) The second regime of $\mathrm{Au}$ intercalation consists of $\mathrm{Au}$ nanoclusters and, therefore, is the one crucial for the giant Rashba effect. It is structurally coherent to the moiré pattern of the continuous $\mathrm{Au}$ monolayer (i. e., to the misfit dislocation loop structure) which is possibly the reason why it has remained unnoticed so far. This second regime comprises two 
types of arrays with large $(\sim 1 \mathrm{~nm})$ and small $(\sim 0.5 \mathrm{~nm})$ Au clusters arranged in $(9.7 \times 9.7)$ and $(3.2 \sqrt{3} \times 3.2 \sqrt{3})$ R30 $0^{\circ}$ superstructures, respectively.

(iii) Our conclusions are robust because we verified them for both types of Ni preparations employed in the literature: a $\mathrm{Ni}(111)$ single crystal and thin $\mathrm{Ni}(111)$ films grown on $\mathrm{W}(110)$.

(iv) Extensive structural calculations performed for intercalated $\mathrm{Au}_{1}$ monomers and $\mathrm{Au}_{3}$ trimers accurately confirm our model in which the enhancement of the Rashba-type spin splitting arises from the attractive interaction between graphene and $\mathrm{Ni}(111)$ in the areas between the Au clusters. The calculations confirm that this interaction pulls the graphene closer to the intercalated $\mathrm{Au}$ cluster and enhances the spin-orbit splitting of the Dirac fermions up to the giant value of $70 \mathrm{meV}$ while keeping the graphene quasi-free-standing with intact Dirac cone.

(v) Our calculations predict in addition to the canonical Rashba-type spin texture a pronounced out-of-plane spin component within a small gap in the Dirac cone of the graphene. The out-of-plane spin is ascribed to spin reorientation due to the structural buckling around the intercalated $\mathrm{Au}$ nanoparticles.

Hence, the concept of nanopatterning does not only enable giant Rashba effects, as demonstrated in the present work, but has also the potential for creation and control of complex spin textures in graphene in three dimensions.

\section{EXPERIMENTAL}

Angle-resolved photoemission measurements were conducted with the endstation ARPES $1^{2}$ (Scienta R8000) at the beamline UE112-PGM2 at BESSY II.

STM measurements have been performed with an Omicron VT STM operated at room temperature. Tungsten tips were prepared by electrochemical etching with subsequent in situ annealing as described elsewhere [59]. The base pressure was better than $3 \times 10^{-10}$ mbar. All STM images were acquired at positive bias on the tip meaning that the occupied states of the sample were probed. Parameters on of the tunneling are specified by the labels placed next to each STM image.

Graphene was prepared by chemical vapor deposition of propylene (at partial pressure of $1 \times 10^{-6}$ mbar) on the Ni substrate held at $\mathrm{T}=800 \mathrm{~K}$. In our experiments, we have tested two common approaches to prepare $\mathrm{Ni}(111)$. The first approach was the use of a $\mathrm{Ni}(111)$ single 
crystal which has been cleaned by repeated cycles of $\mathrm{Ar}^{+}$sputtering at $2 \mathrm{kV}$ and annealing at $\mathrm{T}=1000 \mathrm{~K}$. In the second approach, $\mathrm{Ni}(111)$ was prepared as $\sim 20$ monoatomic layers (ML) deposited on $\mathrm{W}(110)$ and subsequently annealed at $\mathrm{T}=800 \mathrm{~K}$.

Structural properties of Au-intercalated samples were studied systematically as a function of $\mathrm{Au}$ concentrataion. For that purpose, a $\mathrm{Au}$ wedge (from 0 to $1 \mathrm{ML}$ ) was deposited and different sample areas corresponding to different Au concentrations characterized by STM, low-energy electron diffraction (LEED) and photoemission. Deposition rates of the Au evaporator were calibrated by a quartz microbalance and were typically $0.2 \mathrm{ML}$ per minute.

DFT calculations of the band structure of graphene on a continuous Au monolayer have been conducted in the generalized gradient approximation [60] using the full-potential linearized augmented plane-wave method implemented in the FLEUR code (http://www . flapw.de) including spin-orbit interactions. Plane-wave cutoff of $7.37 \AA^{-1}$ and muffintin radii of $0.68 \AA$ for carbon, $1.22 \AA$ for $\mathrm{Au}$ and $1.16 \AA$ for $\mathrm{Ni}$ have been used. For the integration in reciprocal space, $49 k_{\|}$points in the two-dimensional Brillouin zone have been used. The spin-orbit interaction is treated self-consistently as described in Ref. [61]. The continuous Au monolayer was assumed to be pseudomorphic $[p(1 \times 1)]$ to the graphene with a lattice constant of $2.489 \AA$ and to the $\mathrm{Ni}(111)$ substrate. For the calculations, a structural optimization has been conducted by force minimization.

For the investigation of the Au clusters, the VASP package [62] was used for structural and band structure calculations. We have considered small $\mathrm{Au}$ molecules $\left(\mathrm{Au}_{1}\right.$ and $\left.\mathrm{Au}_{3}\right)$ impregnated in a graphene/1 ML Ni slab with lateral dimensions of $(6 \times 6)$ and $(10 \times 10)$ graphene unit cells. The structural optimization of the graphene supercells was done by employing the projector-augmented wave [63] method and a semi-local exchange-correlation potential as implemented by Perdew, Burke and Ernzerhof [60]. During the structural optimization only the $\mathrm{C}$ atoms were allowed to relax until the total energy difference was less than $10^{-4} \mathrm{eV}$ between two consecutive energy minimization steps. Additionally, plane waves with kinetic energies up to $500 \mathrm{eV}$ formed the basis for the expansion of the electronic orbitals, while a $3 \times 3 \times 1 \bar{\Gamma}$-centered k-point mesh and the $\bar{\Gamma}$-point were used to sample the reciprocal space of the $(6 \times 6)$ and $(10 \times 10)$ supercell, respectively. Finally, non-local dispersive van-der-Waals forces were taken into account using the DFT-D2 methodology of Grimme [64], whereas the spin-orbit interaction was included only in the band structure calculation of the most stable of $\mathrm{Au}_{1}$ configurations. 
Visualization of the structures was performed with the VESTA software [65].

\section{Acknowledgments}

This work was supported by SPP 1459 of the Deutsche Forschungsgemeinschaft and by 'Impuls- und Vernetzungsfonds der Helmholtz-Gemeinschaft' under grant no. HRJRG-408 (Helmholtz-Russia Joint Research Group).

[1] M. Batzill, Surf. Sci. Rep. 67, 83 (2012).

[2] X. Liu, C.-Z. Wang, M. Hupalo, H.-Q. Lin, K.-M. Ho, and M. C. Tringides, Crystals 3, 79 (2013).

[3] Y. Dedkov, E. Voloshina, and M. Fonin, Phys. Status Solidi B 252, 451 (2015).

[4] I. Pletikosić, M. Kralj, P. Pervan, R. Brako, J. Coraux, A. T. N’Diaye, C. Busse, and T. Michely, Phys. Rev. Lett. 102, 056808 (2009).

[5] L. Ponomarenko, R. Gorbachev, G. Yu, D. Elias, R. Jalil, A. Patel, A. Mishchenko, A. Mayorov, C. Woods, J. Wallbank, et al., Nature 497, 594 (2013).

[6] S. Rusponi, M. Papagno, P. Moras, S. Vlaic, M. Etzkorn, P. Sheverdyaeva, D. Pacilé, H. Brune, and C. Carbone, Phys. Rev. Lett. 105, 246803 (2010).

[7] J. Sánchez-Barriga, A. Varykhalov, D. Marchenko, M. R. Scholz, and O. Rader, Phys. Rev. B 85, 201413 (2012).

[8] G. Giovannetti, P. Khomyakov, G. Brocks, V. v. Karpan, J. Van den Brink, and P. Kelly, Phys. Rev. Lett. 101, 026803 (2008).

[9] P. Khomyakov, G. Giovannetti, P. Rusu, G. v. Brocks, J. Van den Brink, and P. Kelly, Phys. Rev. B 79, 195425 (2009).

[10] A. Varykhalov, M. R. Scholz, T. K. Kim, and O. Rader, Phys. Rev. B 82, 121101 (2010).

[11] A. J. Marsden, M.-C. Asensio, J. Avila, P. Dudin, A. Barinov, P. Moras, P. M. Sheverdyaeva, T. W. White, I. Maskery, G. Costantini, et al., Phys. Status Solidi RRL 7, 643 (2013).

[12] C. Enderlein, Y. Kim, A. Bostwick, E. Rotenberg, and K. Horn, New J. Phys. 12, 033014 (2010). 
[13] V. Karpan, G. Giovannetti, P. Khomyakov, M. Talanana, A. Starikov, M. Zwierzycki, J. Van Den Brink, G. Brocks, and P. Kelly, Phys. Rev. Lett. 99, 176602 (2007).

[14] O. Rader, A. Varykhalov, J. Sánchez-Barriga, D. Marchenko, A. Rybkin, and A. M. Shikin, Phys. Rev. Lett. 102, 057602 (2009).

[15] D. Marchenko, A. Varykhalov, M. R. Scholz, G. Bihlmayer, E. I. Rashba, A. Rybkin, A. M. Shikin, and O. Rader, Nat. Commun. 3, 1232 (2012).

[16] D. Usachov, A. Fedorov, M. M. Otrokov, A. Chikina, O. Vilkov, A. Petukhov, A. G. Rybkin, Y. M. Koroteev, E. V. Chulkov, V. K. Adamchuk, et al., Nano Lett. 15, 2396 (2015).

[17] D. Marchenko, A. Varykhalov, J. Sánchez-Barriga, O. Rader, C. Carbone, and G. Bihlmayer, Phys. Rev. B 91, 235431 (2015).

[18] A. Y. Tontegode, Prog. Surf. Sci. 38, 201 (1991).

[19] N. R. Gall, E. V. Rut'kov, and A. Y. Tontegode, Int. J. Mod. Phys. B 11, 1865 (1997).

[20] S. Nie, A. L. Walter, N. C. Bartelt, E. Starodub, A. Bostwick, E. Rotenberg, and K. F. McCarty, ACS Nano 5, 2298 (2011).

[21] Y. Gamo, A. Nagashima, M. Wakabayashi, M. Terai, and C. Oshima, Surf. Sci. 374, 61 (1997).

[22] A. Shikin, V. Adamchuk, and K.-H. Rieder, Phys. Solid State 51, 2390 (2009).

[23] A. Varykhalov, D. Marchenko, J. Sánchez-Barriga, M. R. Scholz, B. Verberck, B. Trauzettel, T. O. Wehling, C. Carbone, and O. Rader, Phys. Rev. X 2, 041017 (2012).

[24] A. Grüneis and D. V. Vyalikh, Phys. Rev. B 77, 193401 (2008).

[25] A. Varykhalov and O. Rader, Phys. Rev. B 80, 035437 (2009).

[26] D. Eom, D. Prezzi, K. T. Rim, H. Zhou, M. Lefenfeld, S. Xiao, C. Nuckolls, M. S. Hybertsen, T. F. Heinz, and G. W. Flynn, Nano Lett. 9, 2844 (2009).

[27] N. A. Vinogradov, A. Zakharov, V. Kocevski, J. Rusz, K. Simonov, O. Eriksson, A. Mikkelsen, E. Lundgren, A. Vinogradov, N. Mårtensson, et al., Phys. Rev. Lett. 109, 026101 (2012).

[28] Y. Xue, B. Wu, Y. Guo, L. Huang, L. Jiang, J. Chen, D. Geng, Y. Liu, W. Hu, and G. Yu, Nano Res. 4, 1208 (2011).

[29] D. Marchenko, J. Sánchez-Barriga, M. R. Scholz, O. Rader, and A. Varykhalov, Phys. Rev. B 87, 115426 (2013).

[30] P. W. Sutter, J.-I. Flege, and E. A. Sutter, Nat. Mater. 7, 406 (2008).

[31] E. Sutter, D. Acharya, J. Sadowski, and P. Sutter, Appl. Phys. Lett. 94, 133101 (2009).

[32] J. Sun, Y. Nam, N. Lindvall, M. T. Cole, K. B. Teo, Y. W. Park, and A. Yurgens, Appl. Phys. 
Lett. 104, 152107 (2014).

[33] S. Schumacher, F. Huttmann, M. Petrović, C. Witt, D. F. Förster, C. Vo-Van, J. Coraux, A. J. Martínez-Galera, V. Sessi, I. Vergara, et al., Phys. Rev. B 90, 235437 (2014).

[34] M. Petrović, I. Š. Rakić, S. Runte, C. Busse, J. Sadowski, P. Lazić, I. Pletikosić, Z.-H. Pan, M. Milun, P. Pervan, et al., Nat. Commun. 4 (2013).

[35] J. Mao, L. Huang, Y. Pan, M. Gao, J. He, H. Zhou, H. Guo, Y. Tian, Q. Zou, L. Zhang, et al., Appl. Phys. Lett. 100, 093101 (2012).

[36] O. Vilkov, A. Fedorov, D. Usachov, L. Yashina, A. Generalov, K. Borygina, N. Verbitskiy, A. Grüneis, and D. Vyalikh, Sci. Rep. 3 (2013).

[37] N. I. Verbitskiy, A. V. Fedorov, G. Profeta, A. Stroppa, L. Petaccia, B. Senkovskiy, A. Nefedov, C. Wöll, D. Y. Usachov, D. V. Vyalikh, et al., Sci. Rep. 5, 17700 (2015).

[38] A. Varykhalov, W. Gudat, and O. Rader, Adv. Mater. 22, 3307 (2010).

[39] C. L. Kane and E. J. Mele, Phys. Rev. Lett. 95, 226801 (2005).

[40] F. Kuemmeth, S. Ilani, D. Ralph, and P. McEuen, Nature 452, 448 (2008).

[41] E. I. Rashba, Phys. Rev. B 79, 161409 (2009).

[42] F. Kuemmeth and E. I. Rashba, Phys. Rev. B 80, 241409 (2009).

[43] M. Gmitra, S. Konschuh, C. Ertler, C. Ambrosch-Draxl, and J. Fabian, Phys. Rev. B 80, $235431(2009)$.

[44] A. M. Shikin, A. G. Rybkin, D. Marchenko, A. A. Rybkina, M. R. Scholz, O. Rader, and A. Varykhalov, New J. Phys. 15, 013016 (2013).

[45] E. Zhizhin, A. Varykhalov, A. Rybkin, A. Rybkina, D. Pudikov, D. Marchenko, J. SánchezBarriga, I. Klimovskikh, G. Vladimirov, O. Rader, et al., Carbon 93, 984 (2015).

[46] M. Weser, Y. Rehder, K. Horn, M. Sicot, M. Fonin, A. B. Preobrajenski, E. N. Voloshina, E. Goering, and Y. S. Dedkov, Appl. Phys. Lett. 96, 012504 (2010).

[47] A. Avsar, J. Y. Tan, T. Taychatanapat, J. Balakrishnan, G. Koon, Y. Yeo, J. Lahiri, A. Carvalho, A. Rodin, E. O'Farrell, et al., Nat. Commun. 5 (2014).

[48] A. Varykhalov, J. Sánchez-Barriga, A. M. Shikin, C. Biswas, E. Vescovo, A. Rybkin, D. Marchenko, and O. Rader, Phys. Rev. Lett. 101, 157601 (2008).

[49] A. Varykhalov, J. Sánchez-Barriga, D. Marchenko, P. Hlawenka, P. S. Mandal, and O. Rader, Nat. Commun. 6, 7610 (2015).

[50] P. Rakyta, A. Kormanyos, and J. Cserti, Phys. Rev. B 83, 155439 (2011). 
[51] J. Jacobsen, L. Pleth Nielsen, F. Besenbacher, I. Stensgaard, E. Lægsgaard, T. Rasmussen, K. W. Jacobsen, and J. K. Nørskov, Phys. Rev. Lett. 75, 489 (1995).

[52] S. Kovalenko, V. Yurov, B. Andryushechkin, and K. Eltsov, in Proceedings of SSS TMAS (2013), p. 11.

[53] B. Premlal, M. Cranney, F. Vonau, D. Aubel, D. Casterman, M. M. D. Souza, and L. Simon, Appl. Phys. Lett. 94, 263115 (2009).

[54] G. Li, H. Zhou, L. Pan, Y. Zhang, L. Huang, W. Xu, S. Du, M. Ouyang, A. C. Ferrari, and H.-J. Gao, J. Am. Chem. Soc. 137, 7099 (2015).

[55] L. Petersen and P. Hedegård, Surf. Sci. 459, 49 (2000), URL http://www. sciencedirect. com/science/article/pii/S0039602800004416.

[56] J. I. Pascual, G. Bihlmayer, Y. M. Koroteev, H.-P. Rust, G. Ceballos, M. Hansmann, K. Horn, E. V. Chulkov, S. Blügel, P. M. Echenique, et al., Phys. Rev. Lett. 93, 196802 (2004), URL https://link.aps.org/doi/10.1103/PhysRevLett.93.196802.

[57] P. Roushan, J. Seo, C. V. Parker, Y. S. Hor, D. Hsieh, D. Qian, A. Richardella, M. Z. Hasan, R. J. Cava, and A. Yazdani, Nature 460, 1106 (2009), ISSN 0028-0836, URL http: //www.nature.com/nature/journal/v460/n7259/abs/nature08308.html.

[58] P. Leicht, J. Tesch, S. Bouvron, F. Blumenschein, P. Erler, L. Gragnaniello, and M. Fonin, Phys. Rev. B 90, 241406 (2014), URL https://link.aps.org/doi/10.1103/PhysRevB.90. 241406.

[59] A. Varykhalov, O. Rader, and W. Gudat, Phys. Rev. B 72, 115440 (2005).

[60] J. P. Perdew, K. Burke, and M. Ernzerhof, Phys. Rev. Lett. 77, 3865 (1996).

[61] C. Li, A. J. Freeman, H. Jansen, and C. Fu, Phys. Rev. B 42, 5433 (1990).

[62] G. Kresse and J. Hafner, Phys. Rev. B 47, 558 (1993).

[63] P. E. Blöchl, Phys. Rev. B 50, 17953 (1994).

[64] S. Grimme, J. Comput. Chem. 27, 1787 (2006).

[65] K. Momma and F. Izumi, J. Appl. Crystallogr. 44, 1272 (2011). 


\title{
Supplementary information for "Nanostructural origin of giant Rashba effect in intercalated graphene"
}

\author{
M. Krivenkov, E. Golias, D. Marchenko, J. Sánchez-Barriga, G. Bihlmayer, O. Rader and A. Varykhalov
}

\section{Supplementary Note 1}

We employed as $\mathrm{Ni}(111)$ substrate bulk Ni single crystals as well as epitaxial Ni films on W(110). We do not find a direct connection between the $\mathrm{Au}$ structure and the type of Ni substrates since for both types of Ni substrates we observe simultaneously both $\mathrm{Au}$ phases: the cluster phase and the monolayer phase. Figures 2, 3 and $\mathrm{S} 1$ show Ni(111) single crystal data - the fact that the thin film $\mathrm{Ni}(111) / \mathrm{W}(110)$ supports the same Au structures is seen from Fig. S2. However, it may be that the ratio of the two phases and/or the Rashba splitting is affected by the rotational disorder of the graphene. By rotational disorder of the graphene we mean a phenomenon which is visible as arcs in the LEED pattern and from the mixing of features along $\bar{\Gamma}-\bar{K}$ and $\bar{\Gamma}-\bar{M}$ in the band dispersion measured by ARPES (see, e. g., Fig. S13 of [1]). It is known that this rotational disorder is strongly affected by the preparation conditions (substrate temperature and pressure of hydrocarbon gas). This is very evident from the example of graphene/Co/W(110) [2, 3]. In addition, we find that the rotational disorder tends to be lower for graphene/Ni/W(110) than on the $\mathrm{Ni}(111)$ single crystal. The reason for this is the absence of bulk-derived screw dislocations at the surface and/or a very weak 1D stripe-like pattern with lateral periodicity of $8.5-11 \mathrm{~nm}$, which stems from the relief of strain induced by $\mathrm{W}(110)$. This 1D pattern may assist in better alignment of the graphene domains to the Ni lattice. The presence of the rotational disorder could possibly lead to extra defects at zone boundaries which in turn may provide new intercalation pathways and favor the full $\mathrm{Au}$ monolayer over the cluster phase. Also, the rotation itself could reduce the graphene$\mathrm{Au}$ hybridization and hence the Rashba splitting. The latter scenario has been confirmed for graphene/Ir(111) [4]. The sample with low Rashba splitting of [5] was dominated by such rotational disorder (Fig. S13 of [1]) but at present it cannot be decided which of the listed factors was decisive for the disorder.

[1] D. Marchenko, A. Varykhalov, M. R. Scholz, G. Bihlmayer, E. I. Rashba, A. Rybkin, A. M. Shikin, and O. Rader, Nat. Commun. 3, 1232 (2012).

[2] D. Marchenko, A. Varykhalov, J. Sánchez-Barriga, O. Rader, C. Carbone, and G. Bihlmayer, Phys. Rev. B 91, 235431 (2015).

[3] D. Pacilé, S. Lisi, I. Di Bernardo, M. Papagno, L. Ferrari, M. Pisarra, M. Caputo, S. Mahatha, P. Sheverdyaeva, P. Moras, et al., Phys. Rev. B 90, 195446 (2014).

[4] D. Marchenko, J. Sánchez-Barriga, M. R. Scholz, O. Rader, and A. Varykhalov, Phys. Rev. B 87, 115426 (2013).

[5] A. Varykhalov, J. Sánchez-Barriga, A. M. Shikin, C. Biswas, E. Vescovo, A. Rybkin, D. Marchenko, and O. Rader, Phys. Rev. Lett. 101, 157601 (2008). 

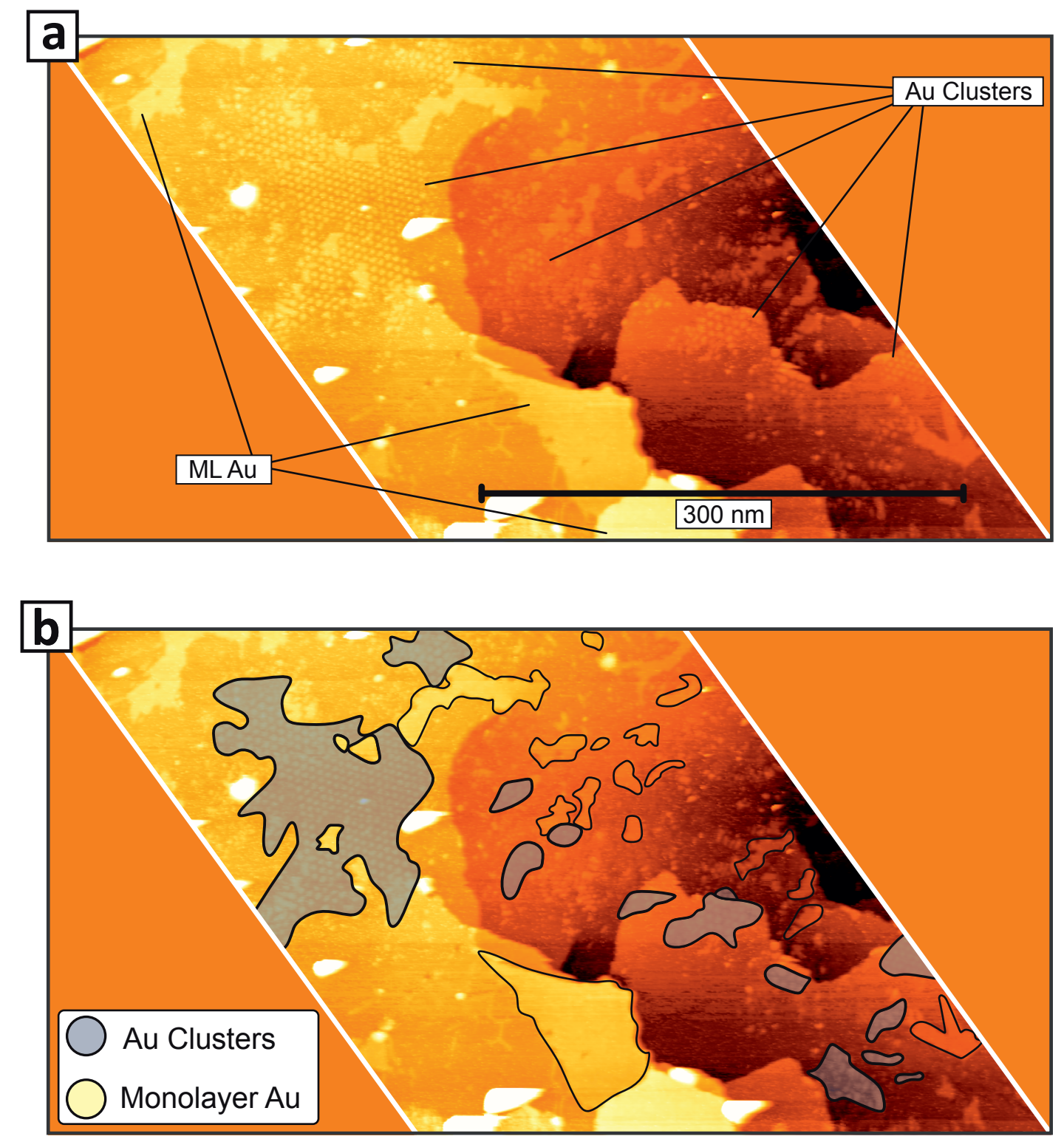

FIG. S1: (a) Large-scale STM data from different sample preparations showing areas of the sample surface with content of clusters of $\sim 50 \%$ in the Au-intercalated areas. (b) The same image as (a), with outlined borders of areas with intercalated clusters (transparent filling) and monolayer $\mathrm{Au}$ (no filling). 

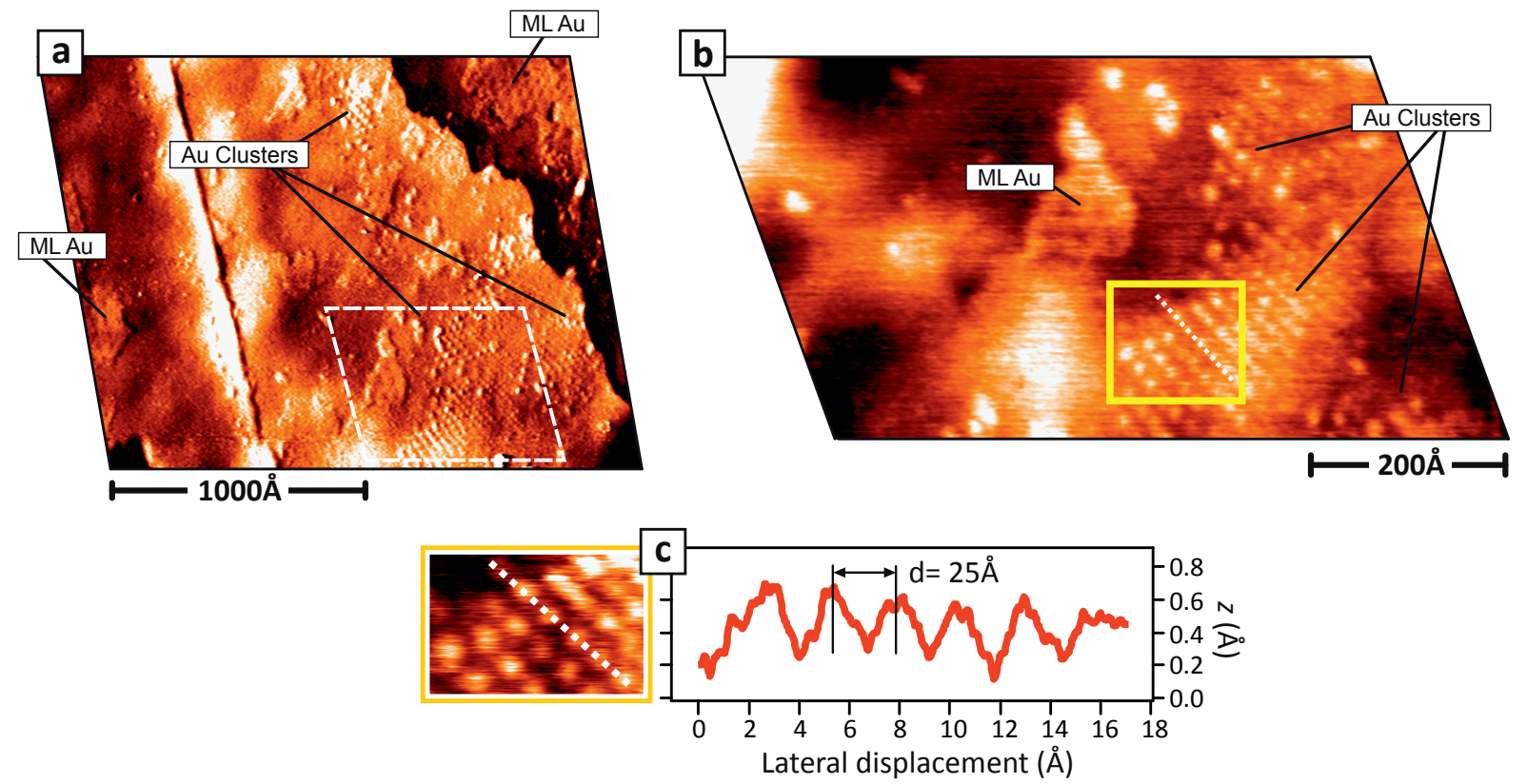

FIG. S2: Observation of a moiré-type (9.7x9.7) continuous Au phase and arrays of Au clusters intercalated under graphene on Ni/W(110). (a) Large scale STM image obtained for low Au concentration; (b) Zoom of the area marked in (a) by white dashed lines; (c) Topographic profile over cluster arrays from (b). It is seen that identically to the case of Au-intercalated graphene on Ni single crystals, both the moiré-type (9.7x9.7) phase and arrays of clusters also appear upon intercalation of Au under graphene grown on Ni films. 